\title{
IN MEMORIAM: HAO WANG
}

\author{
1921-1995
}

Hao Wang, known for his wide-ranging work in mathematical logic and philosophy and for his role as interpreter of and source for the thought of Kurt Gödel, died in New York on May 13, 1995, one week before his 74th birthday. He was born in Jinan, Shandong, China, May 20, 1921. His early studies were in China; he obtained a B.Sc. in mathematics from the National Southwestern Associated University in 1943 and an M.A. in philosophy from Qinghua University in 1945. In 1946 he came to Harvard to study logic and philosophy. From then on his career was in the Western world, although he returned to China a number of times after 1972 and became an honorary professor at Peking University in 1985 and at Qinghua University in 1986.

Wang received his Ph. D. from Harvard in 1948 and was a Junior Fellow of the Society of Fellows at Harvard until 1951. However, he spent much of 1950-51 in Zürich under the auspices of Paul Bernays. Until 1956 he was an assistant professor of philosophy at Harvard, but he was on leave from 1953 to 1955, spending 1953-54 at the Burroughs Corporation and 1954-55 abroad; he was John Locke Lecturer at Oxford in the spring of 1955. In 1956 he became Reader in the Philosophy of Mathematics at Oxford, returning to Harvard in 1961 as Gordon McKay Professor of Mathematical Logic and Applied Mathematics. In 1967 he became a professor at The Rockefeller University and founded a research group in logic. He made Rockefeller an active center, especially of research in set theory. After the group was broken up by the Rockefeller administration in 1976, only Wang remained, even beyond his retirement in 1991.

Among Wang's many contributions to mathematical logic the following might be singled out:

1. The system of set theory in the first edition (1940) of W. V. Quine's Mathematical Logic had been shown to be inconsistent by J. Barkley Rosser. Quine devised what he himself described as a makeshift repair. Wang analyzed the situation more deeply and obtained in [1950] a correct formulation of the intended idea, that the system should add (possibly impredicative) classes to the sets admitted by Quine's well-known system NF. He was able to give a model-theoretic proof of the consistency of the system relative to

The author is indebted to Burton Dreben, Marie L. Grossi, Donald A. Martin, and John McCarthy for assistance. 
NF. What has since been known as ML is Wang's modification, incorporated into the second edition (1951) of Quine's book.

2. Wang undertook in [1954] a predicative foundation of mathematics by constructing a transfinite sequence of ramified systems indexed by ordinals. This work and contemporaneous work of Lorenzen revived the attempt initiated by Hermann Weyl to do analysis predicatively. The time was now ripe for progress in the understanding of predicativity, as Kleene was at the same time developing the theory of hyperarithmetic sets. To answer the question what ordinals should be admitted to index the sequence, Wang made the suggestive proposal that if an ordering of order type $\beta$ is definable in the system $\Sigma_{\alpha}$, then the sequence of systems indexed by $\beta$ should be admissible. This was a principle of autonomous iteration, an idea that played an important part in the analysis of predicativity subsequently, although Wang's particular principle did not accomplish what he thought: All recursive ordinals are admissible, but the orderings definable in $\Sigma_{\alpha}$ for recursive $\alpha$ are hyperarithmetic and so (by a theorem of Spector) give no new ordinals.

3 . Wang began to work with computers early in his career. In the course of a stay at IBM, he obtained in [1960] programs that proved all the theorems of propositional logic in Principia Mathematica in a few minutes, as well as a program that would prove most of the theorems of predicate logic in that work. By using the kind of logical analysis pioneered by Herbrand and Gentzen, he was able to improve substantially on the previous work of Newell, Shaw, and Simon. For this work he was given the first Milestone Award for Automated Theorem Proving in 1983.

4. In collaboration with Andrew S. Kahr and E. F. Moore, Wang proved in 1961 that the AEA case of the decision problem for first-order logic is unsolvable [1962]. This was the only remaining open problem concerning the solvability of a class of prenex formulae without identity defined by a quantifier prefix. In connection with his work on theorem proving he had introduced domino or tiling problems. Given a finite set of unit squares with colored edges, the problem is to determine whether the first quadrant of the Cartesian plane can be covered with copies of these squares so that the colors of abutting edges match (without rotating or reflecting a square), subject to more special constraints. In [1961a] Wang made a connection between problems of this kind and cases related to the AEA case, and then J. R. Büchi used this connection to show that the E\&AEA case is unsolvable. In [1962] it is shown that the solvability of the AEA case is equivalent to that of a "diagonal-constrained" domino problem, where the tiles on the diagonal must belong to a specified subset of the given set, and then this problem is shown unsolvable. Some more general matters are gone into in [1963]. Tiling problems have continued to be explored; cf. [1975]. 
Wang was a prolific writer, and expository writing on logic figures in his early collection [1962a] and in some of his philosophical writings, especially [1974]. However, he wrote only one expository book on logic, [1981], based on lectures given in China and first published in Chinese.

Wang was occupied with philosophy already as a student in China and wrote a number of philosophical essays early in his career, of which probably the most notable is [1961]. His larger-scale philosophical writing, however, begins in the late 1960's. As if by coincidence, an inquiry with Kurt Gödel in 1967 in connection with Wang's work on his introduction [1970] to Skolem's works led to further correspondence and a remarkable series of conversations beginning in 1971 .

Wang's relations with Gödel contributed importantly to [1974], his major statement on the philosophy of mathematics. This work published two letters of Gödel emphasizing the role of his philosophical convictions in his major logical discoveries and also a one-paragraph essay on Turing's analysis of computability. Other parts of the book, such as the important chapter on the concept of set and the chapter on minds and machines, were also influenced by discussion with Gödel. Much of the book, however, hardly reflects Wang's relations with Gödel at all. It is a wide-ranging survey of issues in the foundations of logic and mathematics, giving a great deal of information about the relevant logic and its history. Wang describes his viewpoint as "factualist": Mathematical knowledge is a fact that the philosopher has to make sense of; in particular it is not his role to reject part of mathematics because it fails to meet his standard of philosophical clarity. This viewpoint resembles what other philosophers call naturalism but differs from it in not taking natural science (in particular physics) as a model. The same point of view is also prominent in [1985], a more general philosophical work in which Wang gives expression to his dissatisfaction with the analytic tradition in philosophy in which he had been trained and which continued to exercise a strong hold on him.

Wang's relations with Gödel make his work a unique source concerning Gödel's thought, especially in his later years. [1974] and [1987] already have great value as sources; the forthcoming [199?], completed only a few months before Wang's own death, quotes numerous remarks of Gödel in their conversations. Wang was obviously an interpreter of Gödel as well. His writings on Gödel attempt to capture Gödel's whole intellectual personality. Although he recognized that Gödel's aspirations as a systematic philosopher were only very partially fulfilled, he nonetheless took them seriously, even when the views Gödel expressed were alien to him. No one else has tried so hard to see Gödel whole. But the spectacles with which he saw were very much his own. In particular, his exchanges with Gödel were conversations between two thinkers with synoptic ambitions, one of whom 
sought resources from earlier Western traditions, and the other of whom, however Western his training and career, never ceased to be Chinese.

\section{CITED WRITINGS OF HAO WANG}

[1950] A formal system of logic, Journal of Symbolic Logic, vol. 15, pp. 25-32.

[1954] The formalization of mathematics, Journal of Symbolic Logic, vol. 19, pp. 241-266.

[1960] Toward mechanical mathematics, IBM Journal of Research and Development, vol. 4, pp. $2-22$.

[1961] Process and existence in mathematics, Essays on the foundations of mathematics, dedicated to A. A. Fraenkel, Magnes Press, The Hebrew University of Jerusalem, pp. 328 351 .

[1961a] Proving theorems by pattern recognition, II, Bell System Technical Journal, vol. 40, pp. $1-41$.

[1962] Entscheidungsproblem reduced to the AEA case, with A. S. Kahr and E. F. Moore, Proceedings of the National Academy of Sciences, USA, vol. 48, pp. 365-377.

[1962a] A survey of mathematical logic, Science Press, Peking, also North-Holland, Amsterdam, 1963.

[1963] Dominoes and the AEA case of the decision problem, Proceedings of the Symposium on Mathematical Theory of Automata, Polytechnic Institute of Brooklyn, 1962, Polytechnic Institute, Brooklyn, pp. 23-55.

[1970] A survey of Skolem's work in logic, Th. Skolem, Selected Works in Logic (J. E. Fenstad, editor), Universitetsforlaget, Oslo, pp. 17-52.

[1974] From mathematics to philosophy, Routledge and Kegan Paul, London.

[1975] Notes on a class of tiling problems, Fundamenta Mathematicae, vol. 82, pp. 295-305.

[1981] Popular lectures on mathematical logic, Science Press, Beijing, in Chinese, also Van Nostrand Reinhold, New York, in English.

[1985] Beyond analytic philosophy: Doing justice to what we know, MIT Press, Cambridge, Massachusetts.

[1987] Reflections on Kurt Gödel, MIT Press, Cambridge, Massachusetts.

[199?] A logicaljourney: From Gödel to philosophy, MIT Press, Cambridge, Massachusetts, forthcoming.

Charles Parsons 